\title{
Physicochemical and Biological Properties of Polysaccharides from Dictyophora indusiata Prepared by Different Extraction Techniques
}

\author{
Ding-Tao Wu ${ }^{1,2, *,+}+\mathbb{D}$, Yun-Xuan Zhao ${ }^{2,+}$, Huan Guo ${ }^{2,3}$, Ren-You Gan ${ }^{1,3}{ }^{\mathbb{D}}$, Lian-Xin Peng ${ }^{1}$, Gang Zhao ${ }^{1}$ \\ and Liang Zou ${ }^{1, *}$
}

check for

updates

Citation: Wu, D.-T.; Zhao, Y.-X.; Guo, H.; Gan, R.-Y.; Peng, L.-X.; Zhao, G.;

Zou, L. Physicochemical and

Biological Properties of

Polysaccharides from Dictyophora indusiata Prepared by Different Extraction Techniques. Polymers 2021, 13, 2357. https://doi.org/10.3390/ polym13142357

Academic Editors: Aleksandra Vojvodić Cebin and Draženka Komes

Received: 13 May 2021

Accepted: 2 July 2021

Published: 19 July 2021

Publisher's Note: MDPI stays neutral with regard to jurisdictional claims in published maps and institutional affiliations.

Copyright: (c) 2021 by the authors. Licensee MDPI, Basel, Switzerland. This article is an open access article distributed under the terms and conditions of the Creative Commons Attribution (CC BY) license (https:// creativecommons.org/licenses/by/ $4.0 /)$
1 Key Laboratory of Coarse Cereal Processing, Ministry of Agriculture and Rural Affairs, Sichuan Engineering \& Technology Research Center of Coarse Cereal Industralization, School of Food and Biological Engineering, Chengdu University, Chengdu 610106, China; ganrenyou@caas.cn (R.-Y.G.); penglianxin@cdu.edu.cn (L.-X.P.); zhaogang@cdu.edu.cn (G.Z.)

2 Institute of Food Processing and Safety, College of Food Science, Sichuan Agricultural University, Ya'an 625014, China; zhaoyunxuan0320@163.com (Y.-X.Z.); ghscny@163.com (H.G.)

3 Research Center for Plants and Human Health, Institute of Urban Agriculture, Chinese Academy of Agricultural Sciences, Chengdu 610213, China

* Correspondence: dt_wu@sicau.edu.cn or wudingtao@cdu.edu.cn (D.-T.W.); zouliang@cdu.edu.cn (L.Z.)

+ These authors contributed equally to this work.

Abstract: In this study, different extraction techniques, including traditional hot water extraction (HWE), microwave-assisted extraction (MAE), pressurized assisted extraction (PAE), and ultrasonicassisted extraction (UAE), were used to extract Dictyophora indusiata polysaccharides (DFPs), and their physicochemical and biological properties were compared. Results revealed that extraction yields of D. indusiata polysaccharides prepared by different extraction techniques ranged from $5.62 \%$ to $6.48 \%$. D. indusiata polysaccharides prepared by different extraction techniques possessed similar chemical compositions and monosaccharide compositions, while exhibited different molecular weights $\left(M_{w}\right)$, apparent viscosities, and molar ratios of constituent monosaccharides. In particularly, D. indusiata polysaccharides prepared by HWE (DFP-H) had the highest $M_{w}$ and apparent viscosity among all DFPs, while $D$. indusiata polysaccharides extracted by UAE (DFP-U) possessed the lowest $M_{w}$ and apparent viscosity. In addition, the in vitro antioxidant effects of $D$. indusiata polysaccharides prepared by PAE (DFP-P) and DFP-U were significantly higher than that of others. Indeed, both DFP-P and DFP-H exhibited much higher in vitro binding properties, including fat, cholesterol, and bile acid binding properties, and lipase inhibitory effects than that of $D$. indusiata polysaccharides prepared by MAE (DFP-M) and DFP-U. These findings suggest that the PAE technique has good potential for the preparation of $D$. indusiata polysaccharides with desirable bioactivities for the application in the functional food industry.

Keywords: Dictyophora indusiata polysaccharide; extraction technique; structural properties; antioxidant activity; binding properties

\section{Introduction}

Polysaccharides are considered to be the major bioactive components in edible and medicinal mushrooms, which possess diverse health-promoting effects, such as antioxidant, anti-tumor, anti-inflammatory, immunomodulatory, anti-diabetic, and gut microbiota regulation effects [1-3]. Due to their diverse health-promoting effects, mushroom polysaccharides have been widely utilized for the prevention and treatment of diseases. In recent years, mushroom polysaccharides have also been extensively used in the food industry, such as functional foods, dietary supplements, and edible films for food packaging [1]. For example, Inonotus obliquus polysaccharides are developed as functional and health products for reducing blood fat, removing free radicals, and regulating immune 
function [4]. Lentinus edodes polysaccharides are incorporated in various food/beverage products to obtain fortified functional foods/beverages for enhancing immune function, lowering serum lipid, lowering blood glucose, and regulating gut microbiota [5]. In addition, polysaccharides extracted from Ganoderma lucidum, Grifola frondosa, and Trametes versicolor have been developed as dietary supplements for promoting immune function [1]. At present, the development of mushroom polysaccharides as functional foods has attracted increasing attention in the food industry.

As one of the most popular edible mushrooms in Asian countries, especially in China, Dictyophora indusiata is a saprophytic fungus belonging to the family Phallaceae [6]. It is also regarded as the "queen of mushrooms" because of its pleasing taste and appearance. It is demonstrated that $D$. indusiata possesses excellent nutritional and medicinal functions [7], such as anti-hyperlipidemic [8], anti-tumor [9], anti-inflammatory [7], neuroprotective [10], and hepatic-protective effects [11]. Polysaccharides, the major bioactive constituent of $D$. indusiata, have been plentifully studied due to their low biotoxicities and beneficial effects. Generally, $\beta-1,3$-glucan and galactan are the major bioactive polysaccharides in D. indusiata [6], which have attracted increasing attention in the functional food industry due to their various beneficial effects.

Different extraction techniques can lead to differences in the biological functions of natural polysaccharides [12]. Generally, traditional hot water extraction (HWE) is commonly used as a simple way to prepare polysaccharides, yet the disadvantages of HWE should also be considered, including comparably long operation time, comparably low extraction yields, and high operation temperature [13]. Therefore, several efficient extraction techniques, including microwave-assisted extraction (MAE), ultrasound-assisted extraction (UAE), and pressurized assisted extraction (PAE), have been introduced. Compared with HWE, MAE shows many advantages. It costs less time and solvents, and provides a high extraction yield $[12,14]$. UAE is also an emerging approach with many advantages, such as shortening extraction duration and economizing power consumption $[13,15]$. PAE, another extraction method, can increase the solubility of polysaccharides. PAE can also help infiltrate the solvent into the sample by reducing the surface tension and viscosity [16]. Indeed, although the structural features and biological functions of $D$. indusiata polysaccharides (DFPs) prepared by traditional hot water extraction [17,18], acid extraction [8], and enzyme-assisted extraction [19] have been reported, the physicochemical properties and biological functions of $D$. indusiata polysaccharides prepared by different efficient extraction techniques such as MAE, UAE, and PAE have seldom been compared. Therefore, it is worthwhile to investigate physicochemical and biological properties of DFPs prepared by different extraction techniques, which is beneficial for the improvement of their applications in the functional food industry.

This study aimed to investigate and compare physicochemical properties, in vitro antioxidant activities, and in vitro hypolipidemic activities (in vitro binding properties and inhibitory effects on lipase) of DFPs prepared by HWE, MAE, UAE, and PAE. Results from this work can provide practical and scientific foundations to choose satisfying extraction techniques to produce DFPs with desirable properties for application in the functional food industry.

\section{Materials and Methods}

\subsection{Samples and Chemicals}

The fruiting body of $D$. indusiata was bought from the local supermarket in Ya'an, China. The sample was dried, grounded, and sieved through a 60-mesh screen. The powders were stored at $-20{ }^{\circ} \mathrm{C}$ before use. Carboxymethyl cellulose (CM-cellulose), cholesterol, sodium deoxycholate, sodium taurocholate, sodium glycocholate, sodium cholate, oleic acid, 1-phenyl-3-methyl-5-pyrazolone (PMP), monosaccharide standards, Griess reagent, 2,2-diphenyl-1-(2,4,6-trinitrophenyl) hydrazyl (DPPH), and 2,2'-azinobis(3-ethylbenzothiazoline-6-sulphonic acid) (ABTS) were obtained from Sigma-Aldrich 
(Sigma, St. Louis, MO, USA). A free cholesterol assay kit was obtained from Solarbio (Solarbio, Beijing, China).

\subsection{Preparation of D. Indusiata Polysaccharides (DFPs)}

\subsubsection{Traditional Hot Water Extraction}

Traditional hot water extraction (HWE) was conducted as the method reported in our previous study with small adjustments [6]. Briefly, D. indusiata powders (1.0 g) were added into $35.0 \mathrm{~mL}$ of deionized water, and the polysaccharides were extracted twice at $95^{\circ} \mathrm{C}$ (water bath) for $2.5 \mathrm{~h}$. The water extract was obtained by centrifuging at $4000 \times g$ for $15 \mathrm{~min}$, and four volumes of $95 \%$ ethanol $(v / v)$ were then added and settled overnight. The precipitates were re-dissolved and freeze-dried, and coded as DPF-H. The phenol-sulfuric acid method and Bradford method were used to detect the total polysaccharides and proteins in DPF-H, respectively. A flow chart for the extraction of polysaccharides from D. indusiata was shown in Figure 1.

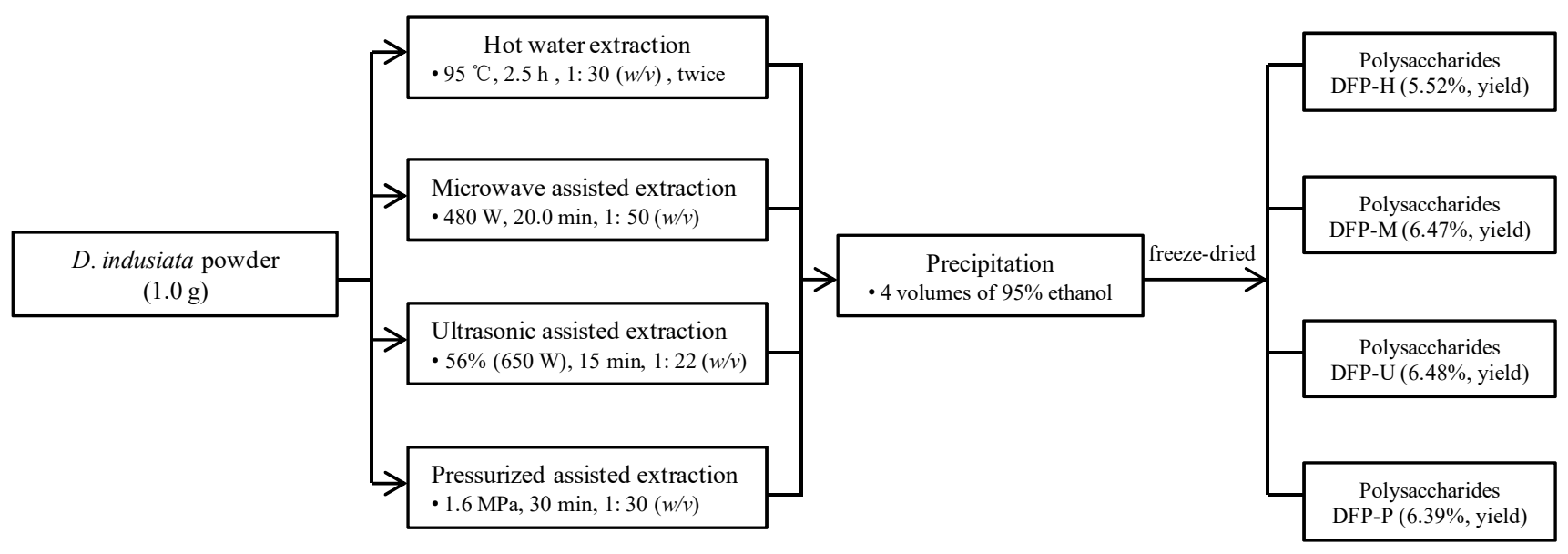

Figure 1. The flow chart for the extraction of polysaccharides from D. indusiata by different techniques. DFP-H, DFP-M, DFP-U, and DFP-P, D. indusiata polysaccharides prepared by hot water extraction, microwave assisted extraction, ultrasonic assisted extraction, and pressurized assisted extraction, respectively.

\subsubsection{Microwave Assisted Extraction}

Microwave-assisted extraction (MAE) was also performed as the method reported in our previous study with minor modifications [20]. Briefly, D. indusiata powders (1.0 g) were added into deionized water $(50.0 \mathrm{~mL})$, and the polysaccharides were extracted with a microwave extractor (MKJ-J1-3, Makewave Company, Qingdao, China) for $20.0 \mathrm{~min}$ at $480 \mathrm{~W}$ and $85^{\circ} \mathrm{C}$. The water extract was obtained by centrifuging at $4000 \times g$ for $15 \mathrm{~min}$, and four volumes of $95 \%$ ethanol $(v / v)$ were added and settled overnight. The precipitates were re-dissolved and freeze-dried, and coded as DPF-M. Afterward, the phenol-sulfuric acid method and Bradford method were used to detect the total polysaccharides and proteins in DPF-M, respectively.

\subsubsection{Ultrasonic Assisted Extraction}

Ultrasonic assisted extraction (UAE) was also conducted as our previous study [6]. Briefly, D. indusiata powders $(1.0 \mathrm{~g})$ were added into deionized water $(22.0 \mathrm{~mL})$, and the polysaccharides were extracted with an Ultrasonic Processor $(650 \mathrm{~W}, 24 \mathrm{kHz}$, Scientz Company, Ningbo, China) for $15.0 \mathrm{~min}$ at room temperature, and ultrasonic amplitude was set at $56 \%$. The water extract was obtained by centrifuging at $4000 \times g$ for $15 \mathrm{~min}$, and four volumes of $95 \%$ ethanol $(v / v)$ were added and settled overnight. The precipitates were re-dissolved and freeze-dried, and coded as DPF-U. Finally, the phenol-sulfuric acid method and Bradford method were used to detect the total polysaccharides and proteins in DPF-U, respectively. 


\subsubsection{Pressurized Assisted Extraction}

Pressurized assisted extraction (PAE) was also operated as our previous study with minor modifications [16]. Briefly, the D. indusiata powders (1.0 g) were added into deionized water $(30.0 \mathrm{~mL})$, and the polysaccharides were extracted with a laboratory-scale highpressure reactor (LEC-300, Shanghai Laibei Scientific Instruments Co., Ltd., Shanghai, China) for $30.0 \mathrm{~min}$ at $1.6 \mathrm{MPa}$ and $55^{\circ} \mathrm{C}$. The water extract was obtained by centrifuging at $4000 \times g$ for $15 \mathrm{~min}$, and 4 volumes of $95 \%$ ethanol $(v / v)$ were added and settled overnight. The precipitates were re-dissolved and freeze-dried, and coded as DPF-P. Finally, the phenol-sulfuric acid method and Bradford method were used to detect the total polysaccharides and proteins in DPF-P, respectively.

\subsection{Physicochemical Characterization of DFPs}

\subsubsection{Determination of Molecular Weights and Constituent Monosaccharides}

The weight-average molecular weights $\left(M_{w}\right)$ of DFPs prepared by different extraction techniques were estimated by size exclusion chromatography coupled with a multi-angle laser light scattering and a refractive index detector (Wyatt Technology Co., Santa Barbara, CA, USA) [21]. A Shodex OHpak SB-806M HQ column ( $300 \mathrm{~mm} \times 8.0 \mathrm{~mm}$, i.d.) was used and set at $30^{\circ} \mathrm{C}$. The Astra software (version 7.1.3, Wyatt Technology Co., Santa Barbara, CA, USA) was utilized for data acquisition and analysis.

Constituent monosaccharides of DFPs were also evaluated by high-performance liquid chromatography followed by our previously established method [20]. Rha, Man, GlcA, GalA, Glc, Gal, Xyl, and Ara were used as standards. The constituent monosaccharides of DFPs were analyzed by an Agilent 1260 series LC system (Agilent Technologies, Palo Alto, CA, USA). The ZORBAX Eclipse XDB-C18 column (4.6 mm $\times 250 \mathrm{~mm}$, i.d. $5 \mu \mathrm{m}$, Agilent Technologies Inc.) was used to separate monosaccharides. The mobile phase was $0.1 \mathrm{M}$ phosphate buffer solution $(\mathrm{pH}=6.7)$ and acetonitrile with a ratio of 83: $17(v / v)$. The flow rate was set at $1.0 \mathrm{~mL} / \mathrm{min}$, and the wavelength of DAD was set at $245 \mathrm{~nm}$.

\subsubsection{Determination of Apparent Viscosities}

The apparent viscosities of DFPs prepared by different extraction techniques were measured by a Discovery Hybrid Rheometer-1 (DHR-1, TA Instruments, New Castle, DE, USA). It was equipped with a parallel steel plate ( $40 \mathrm{~mm}$ diameter, $1.0 \mathrm{~mm}$ gap) [22]. The concentration of each DFP was set at $2.5 \mathrm{mg} / \mathrm{mL}$, and apparent viscosities were measured at 0.1 to $100 \mathrm{~s}^{-1}$. The temperature was set at $25^{\circ} \mathrm{C}$.

\subsubsection{Fourier Transform Infrared (FT-IR) Spectroscopy Analysis}

The FT-IR spectroscopy analysis of DFPs prepared by different extraction techniques was conducted according to our previous study [20]. The Nicolet IS 10 FT-IR (Thermo Fisher Scientific, Waltham, MA, USA) was applied for the analysis of the FT-IR spectra of DFPs, which were recorded in the frequency range of $4000-400 \mathrm{~cm}^{-1}$.

\subsection{Evaluation of In Vitro Antioxidant Activities and Hypolipidemic Activities of DFPs \\ 2.4.1. In Vitro Antioxidant Activities}

The in vitro antioxidant activities of DFPs prepared by different extraction techniques, including DPPH, nitric oxide (NO), and ABTS radical scavenging activities, as well as reducing powers, were evaluated based on our previous study [20]. The NO and DPPH radical scavenging activities were determined at $1.0,2.0,3.0,4.0$, and $5.0 \mathrm{mg} / \mathrm{mL}$, and the BHT $(0.20-1.00 \mathrm{mg} / \mathrm{mL})$ was utilized as positive control. The ABTS radical scavenging activities and reducing power were also determined at $1.0,2.0,3.0,4.0$, and $5.0 \mathrm{mg} / \mathrm{mL}$, and the vitamin $C\left(V_{c}\right)$ was used as positive control. The ABTS radical scavenging activities and reducing power of $V_{c}$ were determined in the concentration range of $0.04-0.12 \mathrm{mg} / \mathrm{mL}$ and $0.10-0.30 \mathrm{mg} / \mathrm{mL}$, respectively. 


\subsubsection{In Vitro Hypolipidemic Activities}

The in vitro binding properties (fat, cholesterol, and bile acid-binding properties) and inhibitory effects against the pancreatic lipase of DFPs prepared by different extraction techniques were measured based on our previous study [21]. The fat binding capacity of DFPs was calculated by the weight of bounded fat per weight of DFPs $(\mathrm{g} / \mathrm{g})$, and CM-cellulose was used as positive control. The cholesterol-binding activity of DFPs was calculated by milligram of binding cholesterol per gram of DPFs $(\mathrm{mg} / \mathrm{g})$, and CM-cellulose was used as positive control. The bile acid-binding activity of DFPs was expressed as percent of blank control (\%), and cholestyramine was used as the positive control. The pancreatic lipase inhibitory effects of DFPs prepared by different extraction techniques were determined at 1.0, 2.0,3.0, 4.0, and $5.0 \mathrm{mg} / \mathrm{mL}$, and the orlistat was used as the positive control.

\subsection{Statistical Analysis}

Each experiment was performed three times, and the final data were expressed as means \pm standard deviations. OriginLab 9.0 (OriginLab Corporation, Northampton, MA, USA) was applied for data analysis. Statistical differences $(p<0.05)$ were compared by one-way analysis of variance (ANOVA) with Duncan's multiple range test.

\section{Results and Discussion}

\subsection{Comparison of Physicochemical Characteristics of DFPs Prepared by Different Techniques}

\subsubsection{Chemical Compositions of DFPs}

Table 1 summarizes the basically chemical compositions and extraction yields of DFPs prepared by HWE, MAE, UAE, and PAE. It can be seen from Table 1 that the extraction yields of DFPs prepared by HWE, MAE, UAE, and PAE ranged from $5.62 \%$ to $6.48 \%$. The extraction yields of DFPs were similar to the result of a previous study using ultrasound assisted extraction [6], but lower than that of an optimized hot water extraction [18] and an optimized enzyme-assisted extraction [19], which might be attributed to different resources of D. indusiata used. The extraction yields of DFP-M, DFP-U, and DFP-P were similar, which were higher than that of DFP-H. In addition, the total polysaccharides in DFPs were also influenced by different extraction techniques, similar to previously reported [16]. The total polysaccharides in DFP-H, DFP-M, DFP-U, and DFP-P were measured to be $83.68 \pm 0.28 \%, 81.19 \pm 0.33 \%, 80.37 \pm 0.29 \%$, and $86.17 \pm 0.38 \%$, respectively, indicating that polysaccharides were the major components in DFPs. A small amount of proteins were determined in DFPs, which ranged from $1.27 \%$ to $3.43 \%$. The contents of total uronic acids in DFPs ranged from $1.94 \%$ to $3.16 \%$. Compared with the HWE, UAE, MAE, and PAE not only saved extraction time, but also led to high extraction yields. In addition, the DFP-P obtained by the PAE method showed the highest content of total polysaccharides among all DFPs. Generally, pressurized assisted extraction can increase the solubility of polysaccharides, and contribute to the solvent infiltrate into the sample by reducing the surface tension and viscosity, thereby increasing the yields of polysaccharides $[16,22]$. The PAE method is worthy of application for the extraction of polysaccharides from $D$. indusiata in the food industry.

\subsubsection{Constituent Monosaccharides, Molecular Weights, and Apparent Viscosities of DFPs}

The monosaccharides, molecular weights, and apparent viscosities are generally to be highly related to the biological activities of natural polysaccharides [23-25]. Thus, the constituent monosaccharides, molecular weights, and apparent viscosities of DFPs prepared by HWE, MAE, UAE, and PAE were measured. Figure $2 \mathrm{~A}$ reveals that the HPLC-UV chromatograms of all DFPs prepared by different extraction techniques were similar. Previous results also indicated that compositional monosaccharides of DFP-H, DFP-M, DFP-U, and DFP-P were Man, Rha, GlcA, Gal, and Glc [11]. 
Table 1. Chemical compositions, molecular weights $\left(M_{w}\right)$, and compositional monosaccharides of DFP-H, DFP-M, DFP-U, and DFP-P.

\begin{tabular}{|c|c|c|c|c|}
\hline Samples & DFP-H & DFP-M & DFP-P & DFP-U \\
\hline Extraction yield (\%) & $5.52 \pm 0.14^{b}$ & $6.47 \pm 0.17^{\mathrm{a}}$ & $6.39 \pm 0.13^{a}$ & $6.48 \pm 0.12^{\mathrm{a}}$ \\
\hline Total polysaccharides (\%) & $83.68 \pm 0.28^{b}$ & $81.19 \pm 0.33^{c}$ & $86.17 \pm 0.38^{a}$ & $80.37 \pm 0.29^{d}$ \\
\hline Proteins $(\%)$ & $2.07 \pm 0.08^{c}$ & $3.43 \pm 0.15^{\mathrm{a}}$ & $2.84 \pm 0.12^{b}$ & $1.27 \pm 0.13^{\mathrm{d}}$ \\
\hline Total uronic acids (\%) & $1.94 \pm 0.07^{\mathrm{c}}$ & $2.82 \pm 0.09^{b}$ & $1.99 \pm 0.09^{c}$ & $3.16 \pm 0.11^{\mathrm{a}}$ \\
\hline \multicolumn{5}{|l|}{$M_{w} \times 10^{4}(\mathrm{Da})$} \\
\hline Fraction 1 & 154.73 & 132.02 & 147.29 & 108.97 \\
\hline Fraction 2 & 12.65 & 13.28 & 9.82 & 10.09 \\
\hline Fraction 3 & 6.68 & 7.80 & 5.91 & 6.11 \\
\hline \multicolumn{5}{|l|}{$M_{w} / M_{n}$} \\
\hline Fraction 1 & 1.94 & 2.01 & 1.96 & 1.64 \\
\hline Fraction 2 & 1.07 & 1.24 & 1.28 & 1.23 \\
\hline Fraction 3 & 1.18 & 1.02 & 1.05 & 1.02 \\
\hline \multicolumn{5}{|c|}{ Monosaccharides and molar ratios } \\
\hline Mannose & 2.68 & 4.19 & 3.30 & 2.68 \\
\hline Rhamnose & 1.00 & 1.00 & 1.00 & 1.00 \\
\hline Glucuronic acid & 0.98 & 0.44 & 0.75 & 0.46 \\
\hline Galactose & 2.13 & 1.74 & 3.59 & 1.24 \\
\hline Glucose & 10.50 & 10.70 & 7.91 & 8.83 \\
\hline
\end{tabular}

DFP-H, DFP-M, DFP-U, and DFP-P are the D. indusiata polysaccharides prepared by HWE, MAE, UAE, and PAE, respectively; Values represent mean \pm standard deviation, and superscripts a-d differ significantly $(p<0.05)$ among extraction yields, total polysaccharides, and total uronic acids of $D$. indusiata polysaccharides prepared by different extraction methods, respectively; Statistical significances were carried out by ANOVA plus post hoc Ducan's test. Fractions 1-3 were the same in Figure 2B.

A
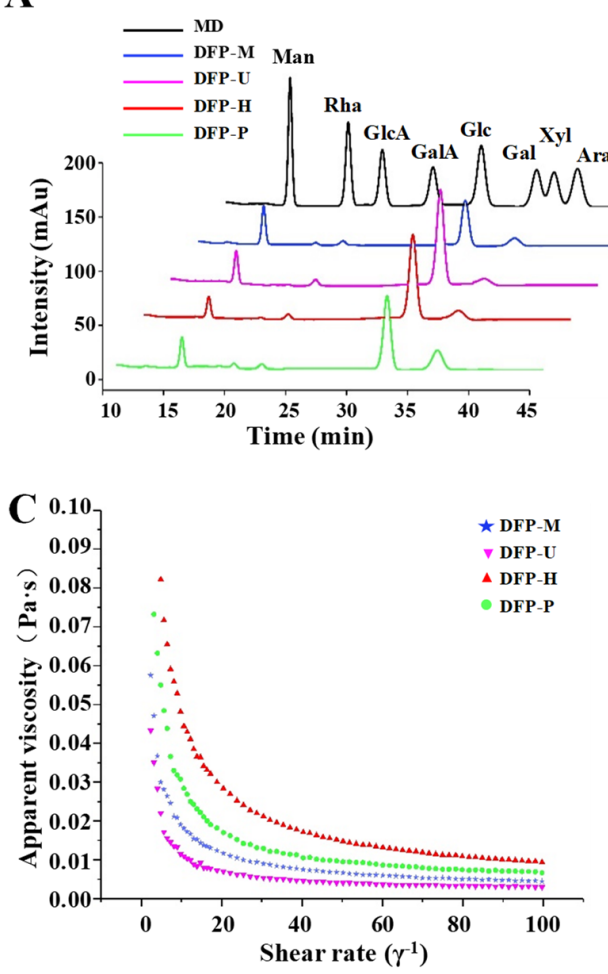
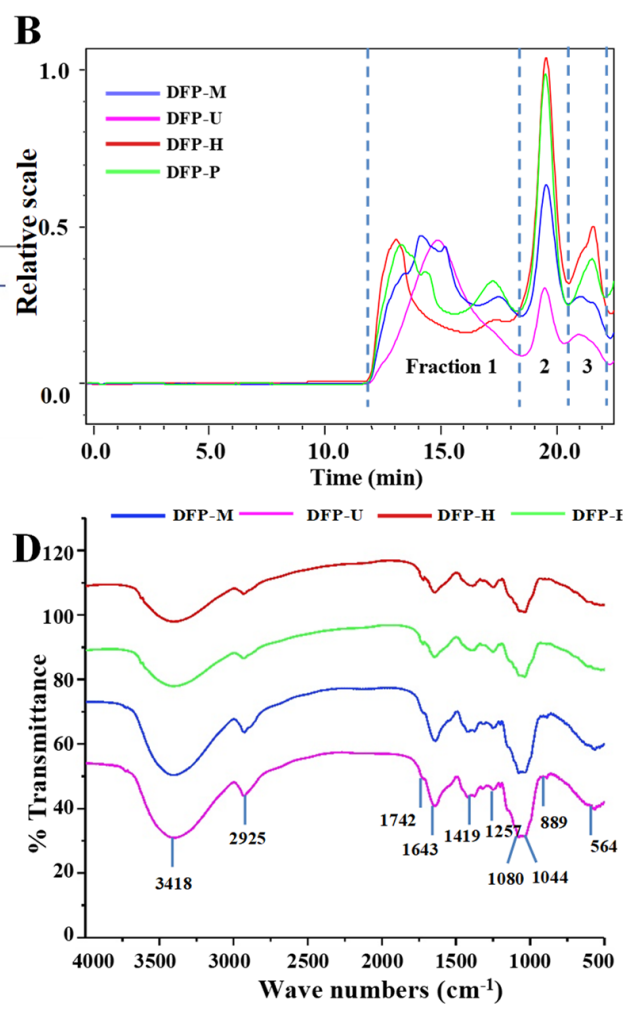

Figure 2. HPLC profiles (A); HPSEC chromatograms (B); apparent viscosities (C) and FT-IR spectra (D) of DFP-H, DFP-M, DFP-U, and DFP-P (D. indusiata polysaccharides prepared by HWE, MAE, UAE, and PAE, respectively); MD, mixed standard of monosaccharides; Man, mannose; Rha, rhamnose; GlcA, glucuronic acid; GalA, galacturonic acid; Glc, glucose; Gal, galactose; Xyl, xylose; Ara, arabinose. $\mathrm{mAu}$, the signal intensity of monosaccharides derivatized with PMP detected by diode array detector. 
The molar ratios of the detected monosaccharides in DFP-H, DFP-M, DFP-U, and DFP-P are shown in Table 1, and the dominant monosaccharide in DFPs was Glc. The types of constituent monosaccharides in DFPs were not influenced by different methods, but their molar ratios were significantly influenced. The differences in constituent monosaccharides of natural polysaccharides obtained from different extraction methods were also reported by other studies $[14,16]$.

Furthermore, Figure 2B displays the HPSEC-RID chromatograms of DFPs prepared by different extraction techniques, and three polysaccharide fractions (1 to 3) were detected. Fraction 1 was determined as the major polysaccharide fraction in DFPs, which obviously degraded during PAE, MAE, and UAE extraction (Figure 2B). Table 1 also shows the molecular weights of polysaccharide fractions (1 to 3) in D. indusiata. The molecular weights of polysaccharide fractions 1 to 3 in DFP-H, DFP-M, DFP-U, and DFP-P are also presented in Table 1, and varied from $108.97 \times 10^{4} \mathrm{Da}$ to $154.73 \times 10^{4} \mathrm{Da}$ (fraction 1 ), $9.82 \times 10^{4} \mathrm{Da}$ to $13.28 \times 10^{4} \mathrm{Da}$ (fraction 2), and $5.91 \times 10^{4} \mathrm{Da}$ to $7.80 \times 10^{4} \mathrm{Da}$ (fraction 3), respectively.

The order of molecular weights of polysaccharide fraction 1 in DFPs was DFP-H > DFP-P > DFP-M > DFP-U, suggesting that different extraction techniques remarkably influenced molecular weights of DFPs. Compared with the molecular weights of DFP$\mathrm{H}$, molecular weights of DFP-P were slightly lower, and DFP-M as well as DFP-U were significantly lower. Results showed that DFPs might degrade during the UAE, MAE, and PAE extraction, respectively. This phenomenon was similar to other studies that molecular weights of natural polysaccharides prepared by MAE and UAE were lower than that of HWE $[15,16,20]$. Furthermore, the polydispersities of fractions 1 to 3 in DFP-H, DFP-M, DFP-U, and DFP-P ranged from 1.64 to 2.01, from 1.07 to 1.28 , and from 1.02 to 1.18, respectively, which were consistent with their HPSEC chromatograms.

Furthermore, the effect of shear rate on apparent viscosities of DFPs prepared by different techniques is shown in Figure 2C. The apparent viscosities of DFP-H, DFP-M, DFP-U, and DFP-P decreased with the increase of the shear rate $\left(0.1-100 \mathrm{~s}^{-1}\right)$. The order of apparent viscosities of DFPs was DFP-H > DFP-P > DFP-M > DFP-U. The apparent viscosities of DFPs were remarkably influenced by extraction techniques. Similar results were reported in other polysaccharides $[16,25,26]$. Indeed, PAE, MAE, and UAE significantly reduced the apparent viscosities of DFPs. This phenomenon was possibly caused by the decrease of their molecular weights and changes of their monosaccharide compositions. The findings suggest that the apparent viscosities of DFP-H, DFP-M, DFP-U, and DFP-P are highly associated with their molecular weights, which is also reported by other studies $[27,28]$.

\subsubsection{FT-IR Spectra of DFPs}

The structural characteristics of DFPs were measured by FT-IR. Figure 2D shows the FT-IR spectra of DFP-H, DFP-M, DFP-U, and DFP-P. DFPs prepared by different extraction techniques showed similar FT-IR spectra, that displayed similar absorption peaks. The broad peaks at $3418 \mathrm{~cm}^{-1}$ and $2925 \mathrm{~cm}^{-1}$ are influenced by the hydroxyl group stretching vibration and $\mathrm{C}-\mathrm{H}$ asymmetric stretching vibration $[8,20]$. The absorption band at $1742 \mathrm{~cm}^{-1}$ is the $\mathrm{C}=\mathrm{O}$ stretching vibration of esterified groups, indicating the presence of uronic acids [15]. The absorption peak at $1643 \mathrm{~cm}^{-1}$ is attributed to the stretching vibration of $\mathrm{C}=\mathrm{O}$, and the absorption peak at $1419 \mathrm{~cm}^{-1}$ is assigned to the $\mathrm{C}-\mathrm{H}$ variable angle vibration [15]. The absorptions in the region of $1000-1200 \mathrm{~cm}^{-1}$ due to stretching vibrations of $\mathrm{C}-\mathrm{OH}$ side groups and the $\mathrm{C}-\mathrm{O}-\mathrm{C}$ glycosidic band [11]. The absorption peak at $889 \mathrm{~cm}^{-1}$ is the typical absorption peak of the $\mathrm{C}-\mathrm{H}$ in $\beta$-linkage, indicating that DFPs contains $\beta$-glucan. The findings were similar to the results of Deng et al. [17]. The results suggested that the types of glycosidic bonds and configurations of polysaccharides extracted from $D$. indusiata were not changed by different extraction techniques, similar to previously reported [15]. 


\subsection{Comparison of In Vitro Antioxidant Activities of DFPs Prepared by Different Extraction Techniques}

The free radical scavenging activity of antioxidants is commonly assessed by the DPPH radical scavenging method [29], therefore, the DPPH radical scavenging capabilities of DFPs extracted by the four methods were measured and compared. The DPPH radical scavenging activities of DFP-H, DFP-M, DFP-U, and DFP-P are shown in Figure 3A, where DFP-H, DFP-M, DFP-U, and DFP-P show noticeable DPPH radical scavenging capabilities.
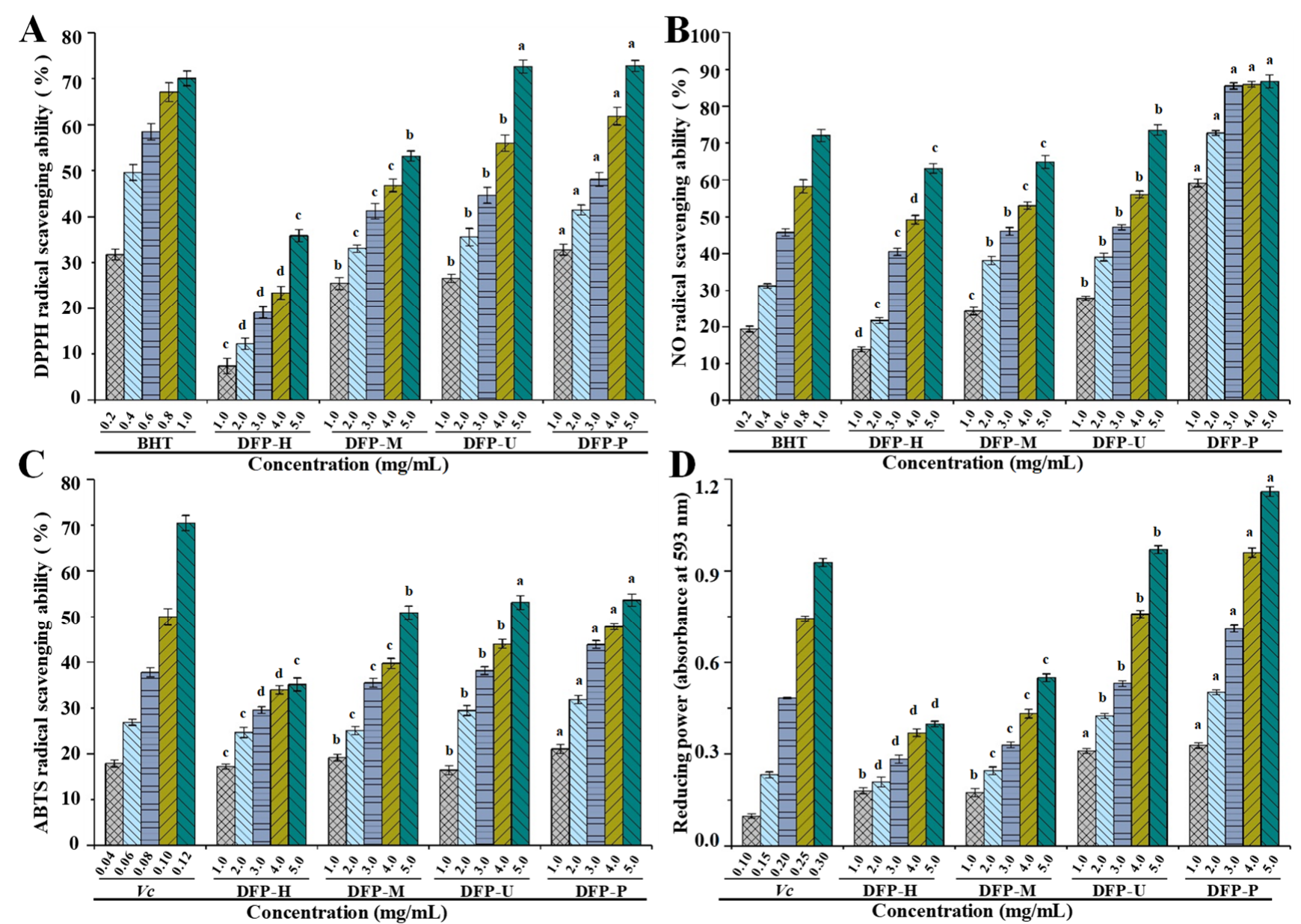

Figure 3. DPPH (A); NO (B) and ABTS (C) radical scavenging activities and reducing powers (D) of DFP-H, DFP-M, DFP-U, and DFP-P (D. indusiata polysaccharides prepared by HWE, MAE, UAE, and PAE, respectively); The error bars are standard deviations; Significant $(p<0.05)$ differences among in vitro antioxidant activities of $D$. indusiata polysaccharides prepared by different extraction methods at the same concentration are shown by data bearing different letters (a-d), respectively; Statistical significances were carried out by ANOVA plus post hoc Duncan's test.

At the concentration of $5.0 \mathrm{mg} / \mathrm{mL}$, the DPPH radical scavenging activities of DFP$\mathrm{H}$, DFP-M, DFP-U, and DFP-P were measured to be $35.88 \pm 1.31 \%, 53.18 \pm 1.12 \%$, $72.59 \pm 1.40 \%$, and $72.77 \pm 1.26 \%$, respectively. In addition, the $\mathrm{IC}_{50}$ value of $\mathrm{DPPH}$ radical scavenging activity of DFP-P was determined to be $2.473 \mathrm{mg} / \mathrm{mL}$. Compared with the $\mathrm{BHT}\left(\mathrm{IC}_{50}=0.418 \mathrm{mg} / \mathrm{mL}\right)$, DFP-P also exerted relatively good DPPH radical scavenging activity. Different extraction methods exhibited obvious influences on the DPPH radical scavenging activities of DFPs, which were similar to other studies $[16,29,30]$. However, Deng et al. [31] found that the DPPH radical scavenging activity of polysaccharides extracted from $D$. indusiata was about $50 \%$ at $1.0 \mathrm{mg} / \mathrm{mL}$. This result was higher than that of DFP-H, DFP-P, DFP-M, and DFP-U. In particular, the DPPH radical scavenging activities of DFP-U and DFP-P were similar, which were higher than those of DFP-M and DFP-H. 
Additionally, as shown in Figure 3B, DFPs also exerted remarkable nitric oxide (NO) radical scavenging activities in a dose-dependent manner. At the concentration of $5.0 \mathrm{mg} / \mathrm{mL}$, the NO radical scavenging activities of DFP-H, DFP-M, DFP-U, and DFP-P were measured to be $63.14 \pm 1.30 \%, 64.91 \pm 1.76 \%, 73.51 \pm 1.43 \%$, and $86.69 \pm 1.80 \%$, respectively. In addition, the $\mathrm{IC}_{50}$ value of NO radical scavenging activity of DFP-P was determined to be $0.698 \mathrm{mg} / \mathrm{mL}$. Compared with the $V_{c}\left(\mathrm{IC}_{50}=0.662 \mathrm{mg} / \mathrm{mL}\right)$, DFP-P also exerted strong NO radical scavenging activity. Different extraction techniques also significantly influenced the NO radical scavenging activities of DFPs, and DFP-P extracted by the PAE technique exhibited obviously stronger NO radical scavenging activities than that of others.

ABTS radical is also commonly applied for the determination of the total antioxidant capacity of various compounds [29]. The ABTS radical scavenging activities of DFP-H, DFP-M, DFP-U, and DFP-P are shown in Figure 3C. Likewise, DFPs prepared by different extraction techniques exhibited obvious ABTS radical scavenging activities with dosedependent manners. At the concentration of $5.0 \mathrm{mg} / \mathrm{mL}$, the ABTS radical scavenging activities of DFP-H, DFP-M, DFP-U, and DFP-P were measured to be $35.11 \pm 1.43 \%$, $50.90 \pm 1.45 \%, 53.12 \pm 1.54 \%$, and $53.62 \pm 1.34 \%$, respectively. Additionally, the ABTS radical scavenging activity of DFP-P $\left(\mathrm{IC}_{50}=4.266 \mathrm{mg} / \mathrm{mL}\right)$ was lower than that of $V_{c}$ $\left(\mathrm{IC}_{50}=0.104 \mathrm{mg} / \mathrm{mL}\right)$. Results showed that ABTS radical scavenging activities of DFP-U and DFP-P were similar, which were higher than that of DFP-M and DFP-H.

For the measurement of the reducing capability, the $\mathrm{Fe}^{3+}-\mathrm{Fe}^{2+}$ transformation in the presence of DFPs prepared by different extraction techniques was investigated by the potassium ferricyanide reduction method [17]. The reducing powers of DFP-H, DFP-M, DFP-U, and DFP-P are shown in Figure 3D. It is seen from Figure 3D that DFP-P also showed the highest reducing power among all samples at the concentrations ranged from 1.0 to $5.0 \mathrm{mg} / \mathrm{mL}(p<0.05)$, followed by DFP-U and DFP-M, and DFP-H displayed the lowest reducing power. At the concentration of $5.0 \mathrm{mg} / \mathrm{mL}$, the absorbances of reducing powers of DFP-H, DFP-M, DFP-U, and DFP-P were measured to be $0.40 \pm 0.02,0.55 \pm 0.02$, $0.97 \pm 0.03$, and $1.16 \pm 0.03$, respectively. The reducing power of DFP-P was lower than that of $V_{c}$. The findings indicated that the reducing powers of DFPs were obviously influenced by different extraction techniques, similar results can also be found in other studies $[20,32,33]$. Studies have shown that the antioxidant activities of natural polysaccharides are associated with their structure features, compositional monosaccharides, and molecular weights [23-25]. Simultaneously, the physicochemical characteristics of $D$. indusiata polysaccharides were influenced by different extraction techniques [34]. In the present study, the higher antioxidant activities observed in DFP-P and DFP-U might be partially attributed to their contents of total polysaccharides, constituent monosaccharides, and molecular weights [35]. Furthermore, it is worth noting that the presence of electrophilic groups like aldehyde or keto can promote the liberation of hydrogen from $\mathrm{O}-\mathrm{H}$ bond, and these groups can also improve the antioxidant activities of natural polysaccharides [36].

\subsection{Comparison of In Vitro Hypolipidemic Activities of DFPs Prepared by Different Extraction Techniques}

\subsubsection{Binding Properties of DFPs}

Numerous complications and hyperlipidemia are reported to be related to the excessive intake of fat and cholesterol, including atherosclerosis, cerebral infarction, cardiovascular diseases, diabetes, hemiplegia, stroke, cancer, and so on [8,37]. Carbohydrates, especially polysaccharides, exhibit obvious abilities to lower blood fat and cholesterol, which may contribute to their hypolipidemic activities [21,23]. Figure 4 shows the in vitro binding properties of DFPs prepared by different extraction techniques. The fat (Figure 4A), cholesterol (Figure 4B), and bile acid (Figure 4C) binding abilities of DFP-H, DFP-M, DFP-U, and DFP-P ranged from $2.37 \pm 0.07$ to $7.17 \pm 0.10 \mathrm{~g} / \mathrm{g}$, from $35.91 \pm 0.74$ to $40.61 \pm 1.09 \mathrm{mg} / \mathrm{g}$, and from $30.45 \pm 0.89 \%$ to $35.24 \pm 1.26 \%$, respectively. 

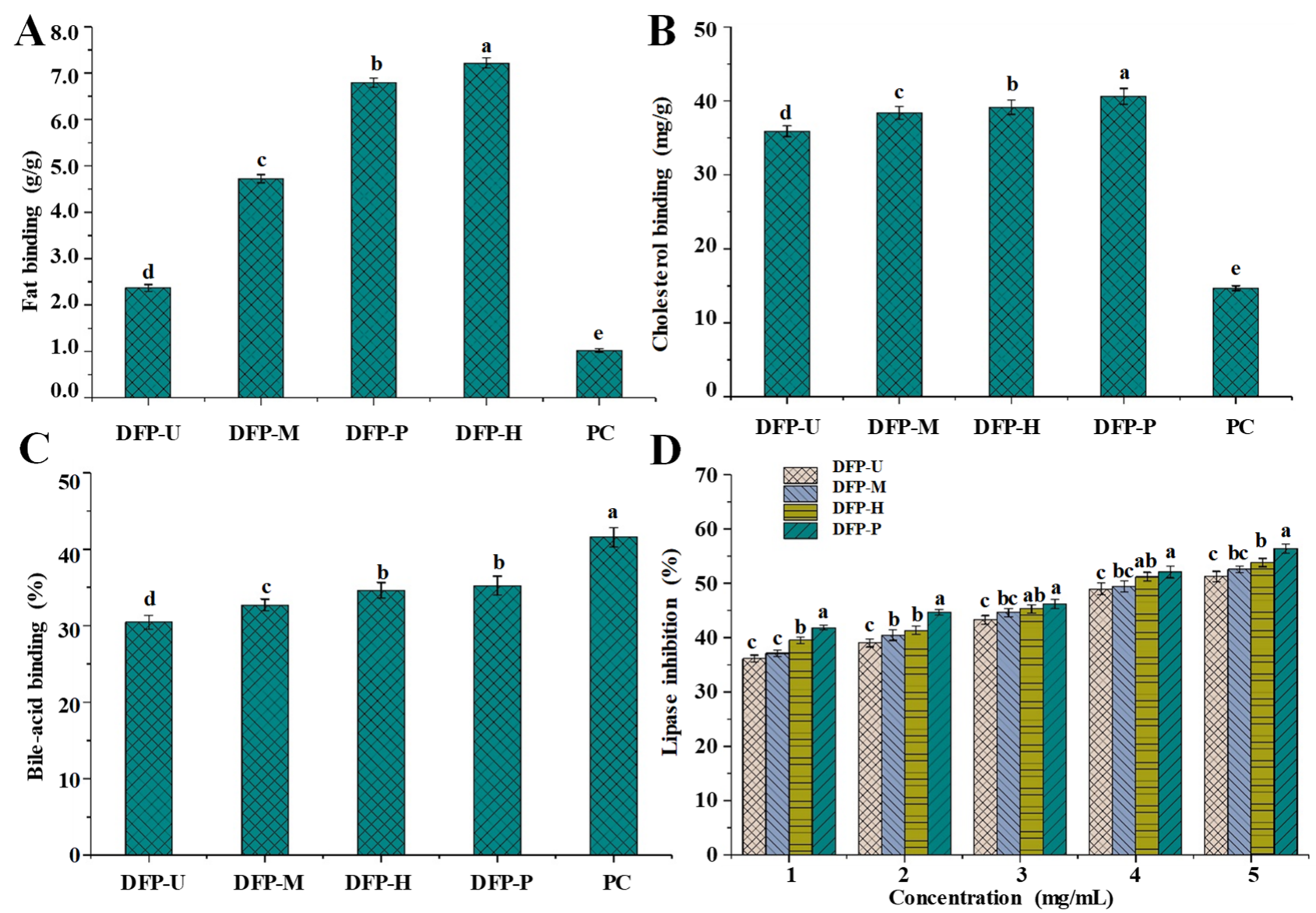

Figure 4. The in vitro fat-binding (A); cholesterol-binding (B); bile-acid binding (C) capacities, and in vitro inhibitory effects on the pancreatic lipase (D) of DFP-H, DFP-M, DFP-U, and DFP-P (D. indusiata polysaccharides extracted by HWE, MAE, UAE, and PAE respectively); PC, positive control; Values represent mean \pm standard deviation, and the letters (a-e) differ significantly $(p<0.05)$ among fat-binding, cholesterol-binding, bile-acid binding capacities, and inhibitory effects on the pancreatic lipase of $D$. indusiata polysaccharides prepared by different extraction methods and the positive control, respectively. Statistical significances were carried out by ANOVA plus post hoc Duncan's test.

Different extraction techniques led to a variation of in vitro binding capacity of DFPs, which was consistent with other studies [16]. Indeed, DFP-P and DFP-H had higher fat binding abilities than that of DFP-U and DFP-M, which were also higher than that of polysaccharides extracted from potatoes peels $(4.398 \pm 0.04 \mathrm{~g} / \mathrm{g})$ [38], but lower than that of polysaccharides extracted from Lilium lancifolium Thunb $(9.25 \pm 0.17 \mathrm{~g} / \mathrm{g}$ ) [39]. Similarly, DFP-P had the highest cholesterol-binding ability among all samples, which was $40.61 \pm 1.09 \mathrm{mg} / \mathrm{g}$. In addition, both DFP-P and DFP-H showed significantly higher bile acid-binding ability, respectively, followed by lower in DFP-M, and the lowest in DFP-U. The results further suggested that the PAE was an outstanding extraction technique because DFP-P had relatively high binding capacities. Several findings have demonstrated that the in vitro binding capacities of polysaccharides are correlated with their apparent viscosities and molecular weights $[16,40]$, which may be due to the high hydrophobic interaction between the fat/cholesterol and polysaccharides [41]. The high molecular weight can reduce the water solubility of DFP-H and DFP-P, resulting in the decrease of their hydrophilicity, which can increase their affinity for the fat/cholesterol by increasing the aggregation on their surface [41]. Due to the solubility in the aqueous condition, $D$. indusiata polysaccharides may have a similar binding mechanism to that of watersoluble fibers. The binding effects of DFP-H and DFP-P were also primarily correlated to their high apparent viscosities. In conclusion, the relatively high binding capacities of DFP-P and DFP-H might be caused by their high molecular weights and apparent 
viscosities. Moreover, compared with the positive controls, DFPs prepared by different techniques exerted better fat and cholesterol binding abilities. Although the bile acidbinding ability of DFPs was lower than that of the positive control, DFPs still showed apparent bile acid-binding capacities. These results confirmed the potential of DFPs used as functional foods/drugs recipes to prevent hyperlipidemia.

\subsubsection{In Vitro Inhibitory Effects of DFPs on the Pancreatic Lipase}

Epidemiological studies have proved that obesity is a risk factor for certain fatal cancers, such as pancreatic ductal adenocarcinoma [42]. Hyperlipidemia is a major risk factor resulting in numerous complications including cardiovascular diseases, atherosclerosis, fatty liver, stroke, diabetes, cerebral infarction, myocardial infarction, hemiplegia, and so on [8,43]. Previous studies have shown that natural polysaccharides exhibit obvious inhibitory effects on the pancreatic lipase [21]. Thus, the present study evaluated the inhibitory effects of DFPs on the pancreatic lipase. The inhibitory activities of DFPs on the pancreatic lipase are summarized in Figure 4D. DFPs prepared by different extraction techniques were able to inhibit the activity of pancreatic lipase, and the inhibitory effects were dose-dependent. The inhibitory effects of DFPs on the pancreatic lipase varied with different extraction techniques. At the concentration of $5.0 \mathrm{mg} / \mathrm{mL}$, the inhibitions on the pancreatic lipase of DFP-H, DFP-M, DFP-U, and DFP$\mathrm{P}$ were measured to be $58.39 \pm 0.98 \%, 52.65 \pm 0.83 \%, 54.67 \pm 1.03 \%$, and $63.20 \pm 1.11 \%$, respectively. Previous studies have shown that natural polysaccharides are capable to limit fat digestion by inhibiting the pancreatic lipase activity, and regulate hyperlipidemia and obesity $[44,45]$. In addition, the inhibitory effects of DFPs on the pancreatic lipase were also positively correlated with their apparent viscosities and molecular weights. In this study, DFPs could inhibit lipase activity to a certain extent might be due to the binding of hydrophobic interaction and electrostatic interaction, resulting in a change in the conformation of lipase, or they could absorb and encapsulate lipase [46]. The highest inhibitory efficiency on the pancreatic lipase was shown by DFP-P, indicating PAE is an efficient method for extracting polysaccharides with relatively high bioactivities. At present, some synthetic drugs, such as statins, have been widely used to treat obesity and hyperlipidemia, but they usually have side effects and are not good for human health [8]. Therefore, there is an urgent need to find new and innovative ways to treat hyperlipidemia. In summary, DFPs, especially DFP-P extracted by PAE, had good potential to be used as functional foods for preventing hyperlipidemia.

\section{Conclusions}

In the present study, different efficient extraction techniques were applied to extract D. indusiata polysaccharides. The results revealed that extraction yields of DFP-M, DFP-U, and DFP-P were similar, which were higher than that of DFP-H. DFPs prepared by different extraction techniques had the same monosaccharide compositions but a slight difference in their molar ratios. In addition, DFP-H had the largest molecular weight and apparent viscosity among all samples, but its antioxidant activity was the weakest among all samples. The strong in vitro antioxidant activity, in vitro binding property, and inhibitory effect on pancreatic lipase were detected in DFP-P. Overall, these findings suggest that the PAE technique can be an efficient method for the preparation of $D$. indusiata polysaccharides with desirable bioactivities for application in the food industry.

Author Contributions: D.-T.W. conceived and designed the experiments; Y.-X.Z. and H.G. performed the experiments; D.-T.W., Y.-X.Z., H.G., L.Z. and R.-Y.G. analyzed the data; G.Z. and L.-X.P. contributed reagents/materials/analysis tools; D.-T.W. wrote the initial draft; D.-T.W. and L.Z. revised the manuscript. All authors have read and agreed to the published version of the manuscript.

Funding: This work was supported by the National Natural Science Foundation of China (grant number 31901690), the Central Public-interest Scientific Institution Basal Research Fund (No. Y2020XK05), and the Scientific Research Foundation of Chengdu University (No. 2081921047).

Institutional Review Board Statement: Not applicable.

Informed Consent Statement: Not applicable. 


\title{
Data Availability Statement: Not applicable.
}

Conflicts of Interest: The authors declare no conflict of interest.

\begin{abstract}
Abbreviations
ABTS, 2,2'-azino-bis (3-ethylbenzothiazoline-6-sulphonic acid; Ara, arabinose; BHT, butylated hydroxytoluene; DPPH, 2,2-diphenyl-1-(2,4,6-trinitrophenyl) hydrazyl; DFPs, Dictyophora indusiata polysaccharides; DFP-H, D. indusiata polysaccharides prepared by hot water extraction; DFP-U, D. indusiata polysaccharides extracted by ultrasonic-assisted extraction; DFP-P, D. indusiata polysaccharides prepared by pressurized assisted extraction; DFP-M, D. indusiata polysaccharides prepared by microwave-assisted extraction; FT-IR, fourier transform infrared spectroscopy; GlcA, glucouronic acid; GalA, galactouronic acid; Glc, glucose; Gal, galactose; HWE, hot water extraction; MAE, microwave-assisted extraction; Man, mannose; $M_{w}$, molecular weights; $N O$, nitric oxide; PAE, pressurized assisted extraction; PMP, 1-phenyl-3-methyl-5-pyrazolone; Rha, rhamnose; UAE, ultrasonicassisted extraction; Xyl, xylose.
\end{abstract}

\section{References}

1. Yan, M.Y.; Belwal, T.; Devkota, H.P.; Luo, Z.S. Trends of utilizing mushroom polysaccharides (MPs) as potent nutraceutical components in food and medicine: A comprehensive review. Trends Food Sci. Tech. 2019, 92, 94-110.

2. Ruthes, A.C.; Smiderle, F.R.; Iacomini, M. Mushroom heteropolysaccharides: A review on their sources, structure and biological effects. Carbohyd. Polym. 2016, 136, 358-375. [CrossRef]

3. Ma, G.X.; Du, H.J.; Hu, Q.H.; Yang, W.J.; Pei, F.; Xiao, H. Health benefits of edible mushroom polysaccharides and associated gut microbiota regulation. Crit. Rev. Food Sci. Nutr. 2021. [CrossRef]

4. Lu, Y.P.; Jia, Y.N.; Xue, Z.H.; Li, N.N.; Liu, J.Y.; Chen, H.X. Recent developments in Inonotus obliquus (Chaga mushroom) polysaccharides: Isolation, Structural characteristics, biological activities and application. Polymers 2021, 13, 1441. [CrossRef]

5. Sheng, K.J.; Wang, C.L.; Chen, B.T.; Kang, M.J.; Wang, M.C.; Liu, K.; Wang, M. Recent advances in polysaccharides from Lentinus edodes (Berk.): Isolation, structures and bioactivities. Food Chem. 2021, 358, 129883. [CrossRef]

6. Fu, Y.; Lin, S.; Lu, M.; Wei, S.Y.; Zhou, J.; Zhao, L.; Zhang, Q.; Lin, D.R.; Liu, Y.T.; Chen, H.; et al. Quantitative evaluation of ultrasound-assisted extraction of 1,3- $\beta$-glucans from Dictyophora indusiata using an improved fluorometric assay. Polymers 2019, 11, 864. [CrossRef]

7. Wang, Y.; Lai, L.; Teng, L.; Li, Y.; Cheng, J.; Chen, J.; Deng, C. Mechanism of the anti-inflammatory activity by a polysaccharide from Dictyophora indusiata in lipopolysaccharide-stimulated macrophages. Int. J. Biol. Macromol. 2019, 126, 1158-1166. [CrossRef]

8. Wang, W.; Song, X.; Gao, Z.; Zhao, H.; Wang, X.; Liu, M.; Jia, L. Anti-hyperlipidemic, antioxidant and organic protection effects of acidic-extractable polysaccharides from Dictyophora indusiata. Int. J. Biol. Macromol. 2019, 129, 281-292. [CrossRef]

9. Deng, C.; Fu, H.; Teng, L.; Hu, Z.; Xu, X.; Chen, J.; Ren, T. Anti-tumor activity of the regenerated triple-helical polysaccharide from Dictyophora indusiata. Int. J. Biol. Macromol. 2013, 61, 453-458. [CrossRef]

10. Zhang, J.; Shi, R.; Li, H.; Xiang, Y.; Xiao, L.; Hu, M.; Ma, F.; Ma, C.W.; Huang, Z. Antioxidant and neuroprotective effects of Dictyophora indusiata polysaccharide in Caenorhabditis elegans. J. Ethnopharmacol. 2016, 192, 413-422. [CrossRef]

11. Wang, W.; Song, X.; Zhang, J.; Li, H.; Liu, M.; Gao, Z.; Wang, X.; Jia, L. Antioxidation, hepatic- and renal-protection of water-extractable polysaccharides by Dictyophora indusiata on obese mice. Int. J. Biol. Macromol. 2019, 134, 290-301. [CrossRef]

12. Shang, H.; Chen, S.; Li, R.; Zhou, H.; Wu, H.; Song, H. Influences of extraction methods on physicochemical characteristics and activities of Astragalus cicer L. Polysaccharides. Process Biochem. 2018, 73, 220-227. [CrossRef]

13. Kang, Q.; Chen, S.; Li, S.; Wang, B.; Liu, X.; Hao, L.; Lu, J. Comparison on characterization and antioxidant activity of polysaccharides from Ganoderma lucidum by ultrasound and conventional extraction. Int. J. Biol. Macromol. 2019, 124, 1137-1144. [CrossRef] [PubMed]

14. He, L.; Yan, X.; Liang, J.; Li, S.; He, H.; Xiong, Q.; Lai, X.; Hou, S.; Huang, S. Comparison of different extraction methods for polysaccharides from Dendrobium officinale stem. Carbohyd. Polym. 2018, 198, 101-108. [CrossRef] [PubMed]

15. Chen, G.; Fang, C.; Ran, C.; Tan, Y.; Yu, Q.; Kan, J. Comparison of different extraction methods for polysaccharides from bamboo shoots (Chimonobambusa quadrangularis) processing by-products. Int. J. Biol. Macromol. 2019, 130, 903-914. [CrossRef] [PubMed]

16. Yuan, Q.; Lin, S.; Fu, Y.; Nie, X.R.; Liu, W.; Su, Y.; Han, Q.H.; Zhao, L.; Zhang, Q.; Lin, D.R.; et al. Effects of extraction methods on the physicochemical characteristics and biological activities of polysaccharides from okra (Abelmoschus esculentus). Int. J. Biol. Macromol. 2019, 127, 178-186. [CrossRef]

17. Deng, C.; Hu, Z.; Fu, H.; Hu, M.; Xu, X.; Chen, J. Chemical analysis and antioxidant activity in vitro of a $\beta$-D-glucan isolated from Dictyophora indusiata. Int. J. Biol. Macromol. 2012, 51, 70-75. [CrossRef]

18. Liu, X.; Chen, Y.; Wu, L.; Wu, X.; Huang, Y.; Liu, B. Optimization of polysaccharides extraction from Dictyophora indusiata and determination of its antioxidant activity. Int. J. Biol. Macromol. 2017, 103, 175-181. [CrossRef] [PubMed] 
19. Wu, S.; Gong, G.; Wang, Y.; Li, F.; Jia, S.; Qin, F.; Ren, H.; Liu, Y. Response surface optimization of enzyme-assisted extraction polysaccharides from Dictyophora indusiata. Int. J. Biol. Macromol. 2013, 61, 63-68. [CrossRef]

20. Guo, H.; Yuan, Q.; Fu, Y.; Liu, W.; Su, Y.H.; Liu, H.; Wu, C.Y.; Zhao, L.; Zhang, Q.; Lin, D.R.; et al. Extraction optimization and effects of extraction methods on the chemical structures and antioxidant activities of polysaccharides from Snow Chrysanthemum (Coreopsis Tinctoria). Polymers 2019, 11, 215. [CrossRef]

21. Guo, H.; Li, H.Y.; Liu, L.; Wu, C.Y.; Liu, H.; Zhao, L.; Zhang, Q.; Liu, Y.T.; Li, S.Q.; Qin, W.; et al. Effects of sulfated modification on the physicochemical properties and biological activities of $\beta$-glucans from Qingke (Tibetan hulless barley). Int. J. Biol. Macromol. 2019, 141, 41-50. [CrossRef] [PubMed]

22. Plaza, M.; Turner, C. Pressurized hot water extraction of bioactives. TrAC Trend Anal. Chem. 2015, 71, 39-54. [CrossRef]

23. Nie, X.R.; Li, H.Y.; Du, G.; Lin, S.; Hu, R.; Li, H.Y.; Zhao, L.; Zhang, Q.; Chen, H.; Wu, D.T.; et al. Structural characteristics, rheological properties, and biological activities of polysaccharides from different cultivars of okra (Abelmoschus esculentus) collected in China. Int. J. Biol. Macromol. 2019, 139, 459-467. [CrossRef]

24. Shah, A.; Gani, A.; Masoodi, F.A.; Wani, S.M.; Ashwar, B.A. Structural, rheological and nutraceutical potential of $\beta$-glucan from barley and oat. Bioact. Carbohydr. Diet. Fibre 2017, 10, 10-16. [CrossRef]

25. Zhu, D.Y.; Ma, Y.L.; Thakur, K.; Wang, C.H.; Wang, H.; Ren, Y.F.; Zhang, J.G.; Wei, Z.J. Effects of extraction methods on the rheological properties of polysaccharides from onion (Allium cepa L.). Int. J. Biol. Macromol. 2018, 112, 22-32. [CrossRef]

26. Yan, J.K.; Wu, L.X.; Cai, W.D.; Xiao, G.S.; Duan, Y.; Zhang, H. Subcritical water extraction-based methods affect the physicochemical and functional properties of soluble dietary fibers from wheat bran. Food Chem. 2019, 298, 124987. [CrossRef]

27. Simas-Tosin, F.F.; Barraza, R.R.; Petkowicz, C.L.O.; Silveira, J.L.M.; Sassaki, G.L.; Santos, E.M.R.; Gorin, P.A.J.; Iacomini, M. Rheological and structural characteristics of peach tree gum exudate. Food Hydrocolloid. 2010, 24, 486-493. [CrossRef]

28. Yuan, Q.; He, Y.; Xiang, P.Y.; Huang, Y.J.; Cao, Z.W.; Shen, S.W.; Zhao, L.; Zhang, Q.; Qin, W.; Wu, D.T. Influences of different drying methods on the structural characteristics and multiple bioactivities of polysaccharides from okra (Abelmoschus esculentus). Int. J. Biol. Macromol. 2020, 147, 1053-1063. [CrossRef]

29. Hu, Z.; Wang, P.; Zhou, H.; Li, Y. Extraction, characterization and in vitro antioxidant activity of polysaccharides from Carex meyeriana Kunth using different methods. Int. J. Biol. Macromol. 2018, 120, 2155-2164. [CrossRef]

30. Dong, H.; Lin, S.; Zhang, Q.; Chen, H.; Lan, W.; Li, H.; He, J.; Qin, W. Effect of extraction methods on the properties and antioxidant activities of Chuanminshen violaceum polysaccharides. Int. J. Biol. Macromol. 2016, 93, 179-185. [CrossRef]

31. Deng, C.; Xu, J.; Fu, H.; Chen, J.; Xu, X. Characterization, antioxidant and cytotoxic activity of sulfated derivatives of a water-insoluble polysaccharides from Dictyophora indusiata. Mol. Med. Rep. 2015, 11, 2991-2998. [CrossRef]

32. Jia, S.; Li, F.; Liu, Y.; Ren, H.; Gong, G.; Wang, Y.; Wu, S. Effects of extraction methods on the antioxidant activities of polysaccharides from Agaricus blazei Murrill. Int. J. Biol. Macromol. 2013, 62, 66-69. [CrossRef]

33. Yan, Y.; Li, X.; Wan, M.; Chen, J.; Li, S.; Cao, M.; Zhang, D. Effect of extraction methods on property and bioactivity of water-soluble polysaccharides from Amomum villosum. Carbohyd. Polym. 2015, 117, 632-635. [CrossRef] [PubMed]

34. Wang, Y.X.; Shi, X.; Yin, J.Y.; Nie, S.P. Bioactive polysaccharide from edible Dictyophora spp.: Extraction, purification, structural features and bioactivities. Bioact. Carbohyd. Diet. Fibre 2018, 14, 25-32. [CrossRef]

35. Cheung, Y.C.; Siu, K.C.; Liu, Y.S.; Wu, J.Y. Molecular properties and antioxidant activities of polysaccharide-protein complexes from selected mushrooms by ultrasound-assisted extraction. Process Biochem. 2012, 47, 892-895. [CrossRef]

36. Wang, J.; Hu, S.; Nie, S.; Yu, Q.; Xie, M. Reviews on mechanisms of in vitro antioxidant activity of polysaccharides. Oxid. Med. Cell. Longev. 2016, 2016, 5692852. [CrossRef]

37. Fu, Y.; Yuan, Q.; Lin, S.; Liu, W.; Du, G.; Zhao, L.; Zhang, Q.; Lin, D.R.; Liu, Y.T.; Qin, W.; et al. Physicochemical characteristics and biological activities of polysaccharides from the leaves of different loquat (Eriobotrya japonica) cultivars. Int. J. Biol. Macromol. 2019, 135, 274-281. [CrossRef]

38. Jeddou, K.B.; Chaari, F.; Maktouf, S.; Nouri-Ellouz, O.; Helbert, C.B.; Ghorbel, R.E. Structural, functional, and antioxidant properties of water-soluble polysaccharides from potatoes peels. Food Chem. 2016, 205, 97-105. [CrossRef] [PubMed]

39. Gao, J.; Zhang, T.; Jin, Z.Y.; Xu, X.M.; Wang, J.H.; Zha, X.Q.; Chen, H.Q. Structural characterisation, physicochemical properties and antioxidant activity of polysaccharide from Lilium lancifolium Thunb. Food Chem. 2015, 169, 430-438. [CrossRef] [PubMed]

40. Lee, S.H.; Jang, G.Y.; Kim, M.Y.; Hwang, I.G.; Kim, H.Y.; Woo, K.S.; Lee, M.J.; Kim, T.J.; Lee, J.; Jeong, H.S. Physicochemical and in vitro binding properties of barley $\beta$-glucan treated with hydrogen peroxide. Food Chem. 2016, 192, 729-735. [CrossRef] [PubMed]

41. Xu, W.; Mohan, A.; Pitts, N.L.; Udenigwe, C.; Mason, B. Bile acid-binding capacity of lobster shell-derived chitin, chitosan and chitooligosaccharides. Food Biosci. 2020, 33, 100476. [CrossRef]

42. Eibl, G.; Cruz-Monserrate, Z.; Korc, M.; Petrov, M.S.; Goodarzi, M.O.; Fisher, W.E.; Habtezion, A.; Lugea, A.; Pandol, S.J.; Hart, P.A.; et al. Diabetes mellitus and obesity as risk factors for pancreatic cancer. J. Acad. Nutr. Diet. 2018, 118, 555-567. [CrossRef] [PubMed]

43. Chen, Y.L.; Xiao, C.H.; Hu, Z.X.; Liu, X.S.; Liu, Z.; Zhang, W.N.; Zhao, X.J. Dynamic lipid profile of hyperlipidemia mice. J. Chromatogr. B 2017, 1055-1056, 165-171. [CrossRef]

44. Aguilera-Angel, E.Y.; Espinal-Ruiz, M.; Narváez-Cuenca, C.E. Pectic polysaccharides with different structural characteristics as inhibitors of pancreatic lipase. Food Hydrocolloid. 2018, 83, 229-238. [CrossRef] 
45. Jin, Q.; Yu, H.; Wang, X.; Li, K.; Li, P. Effect of the molecular weight of water-soluble chitosan on its fat-/cholesterol-binding capacities and inhibitory activities to pancreatic lipase. Peer]. 2017, 5, e3279. [CrossRef]

46. Xue, Z.H.; Gao, X.D.; Jia, Y.N.; Wang, Y.J.; Lu, Y.P.; Zhang, M.; Panichayupakaranant, P.; Chen, H.X. Structure characterization of high molecular weight soluble dietary fiber from mushroom Lentinula edodes (Berk.) Pegler and its interaction mechanism with pancreatic lipase and bile salts. Int. J. Biol. Macromol. 2020, 153, 1281-1290. [CrossRef] [PubMed] 